\title{
Rhizobium radiobacter Endophthalmitis following Intravitreal Ranibizumab Injection
}

\author{
Lavnish Joshi $^{\mathrm{a}, \mathrm{b}} \quad$ Jiten Morarji ${ }^{\mathrm{a}}$ Oren Tomkins-Netzer ${ }^{\mathrm{a}}$ \\ Sue Lightman ${ }^{a, b}$ Simon R.J. Taylor ${ }^{a, c}$
}

${ }^{a}$ Royal Surrey County Hospital NHS Foundation Trust, Guildford, ${ }^{b}$ UCL Institute of Ophthalmology, and 'Division of Immunology and Inflammation, Faculty of Medicine, Imperial College London, London, UK

\section{Key Words}

Rhizobium radiobacter - Gram-negative organism · Intravitreal injection ·

Endophthalmitis

Abstract

We present the first reported case of acute endophthalmitis due to Rhizobium radiobacter after an intravitreal injection of ranibizumab for neovascular age-related macular degeneration.

\section{Introduction}

We present the first reported case of acute endophthalmitis due to Rhizobium radiobacter after an intravitreal injection of ranibizumab for neovascular age-related macular degeneration (AMD).

\section{Case Report}

A 78-year-old lady presented with a one day history of reduced vision, redness and pain in the right eye, seven days after an intravitreal injection of ranibizumab to her right eye for neovascular AMD. Her intravitreal injection had been performed following a standard protocol which included the use of a speculum, but $0.05 \%$ chlorhexidine (Medlock Medical Ltd, UK) rather than $5 \%$ povidone iodine had been instilled into the fornix for three minutes prior to injection, as she had been recorded as developing an 'allergic reaction' to iodine following previous use.

Simon R.J. Taylor, MA, PhD, FRCOphth, Senior Lecturer and Consultant Ophthalmic Surgeon
Imperial College London Faculty of Medicine, Room 5N8B 5th Floor Commonwealth Building, Hammersmith Hospital London W12 ONN (UK)

E-Mail s.r.taylor@imperial.ac.uk 
On examination, her visual acuity was hand movements, down from 20/80 pre-injection. She had circumcorneal injection, a 1-mm hypopyon, a moderate nuclear sclerotic cataract, $3+$ vitreous haze

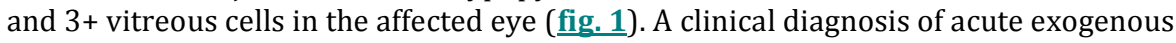
endophthalmitis was made, and she underwent a standard management plan which included anterior chamber and vitreous needle taps, followed by an intravitreal injection of vancomycin $2 \mathrm{mg}$ in $0.1 \mathrm{ml}$ and amikacin $0.4 \mathrm{mg}$ in $0.1 \mathrm{ml}$. She was discharged on topical dexamethasone and chloramphenicol and oral moxifloxacin [1]. Initial Gram stain did not reveal fungal infection, but she was not considered suitable for oral corticosteroids in view of her age and systemic comorbidities (poorly controlled hypertension and well controlled type two diabetes mellitus). At her 48-h review, her eye was pain-free and the hypopyon had resolved.

Culture of the vitreous biopsy specimen revealed the Gram-negative organism Rhizobium radiobacter strain. Antibiotic sensitivity testing showed resistance to ceftazidime, but susceptibility to ciprofloxacin, amikacin and vancomycin. One month after the injection, her visual acuity had improved to 20/100, with no evidence of persistent inflammation. Three months after injection, her visual acuity remained at 20/100.

\section{Discussion}

The incidence of endophthalmitis cases after intravitreal anti-VEGF agents is estimated to be $0.049 \%$, with most isolates being commensals such as coagulasenegative Staphylococcus or Streptococcus viridans [2]. Rhizobium radiobacter has not previously been reported to be a causative organism following intravitreal injection, but its presentation appears no different from other causative organisms. However, its source in our patient is unclear, as it is an organism predominantly found in soil. A few cases have been reported in which it has caused both acute and chronic endophthalmitis following cataract surgery [3], and it was hypothesised in these cases that infection occurred as a result of conjunctival inoculation following patient contact with soil, but our patient reported no gardening activities following her intravitreal injection, suggesting that the organism was already present in the conjunctival sac.

The use of chlorhexidine rather than povidone iodine prior to injection was based on a presumed allergy. However, this is more likely to have represented conjunctival irritation than a true allergy. Chlorhexidine $0.05 \%$ is as effective as $4 \%$ povidone iodine at reducing conjunctival flora in general [4], but there have been concerns that some organisms may be resistant to chlorhexidine [5]. However, this remains controversial, and meta-analysis of its use in other surgical situations has suggested it to be superior to povidone iodine [6]. It is possible that resistance to chlorhexidine might be acquired in certain situations [7], but this patient had previously received only povidone iodine. Lower concentrations of povidone iodine, e.g. $1 \%$, can be used to reduce irritation in susceptible patients, but are considered less effective in patients with higher initial conjunctival bacterial loads [8].

\section{Financial Support}

S.R.J.T. was supported by the UK National Institute of Health Research. This work was supported by the Imperial NIHR Comprehensive Biomedical Research Centre. The sponsor or funding organization had no role in the design or conduct of this research. The authors report no conflicts of interest. This study was approved by the Royal Surrey County Hospital R\&D Department (12DEV0010). 


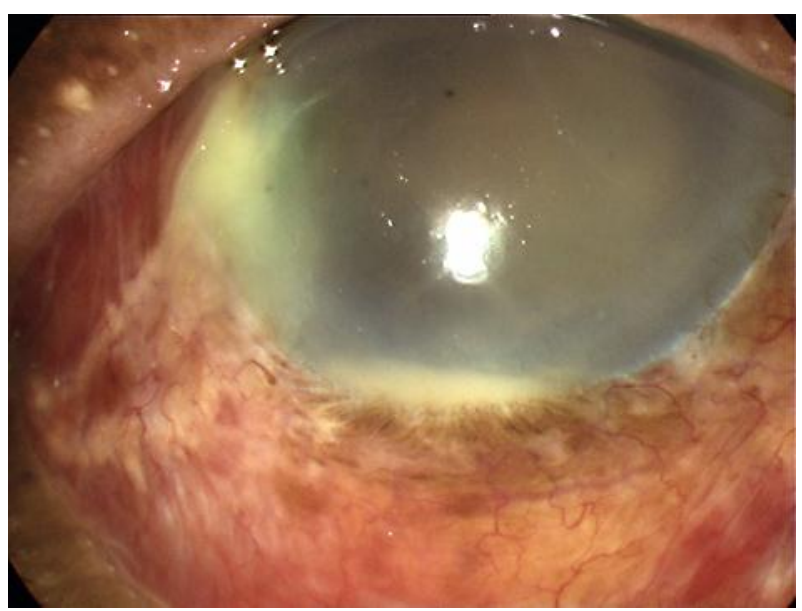

Fig. 1. The patient presented with a clinical picture consistent with endophthalmitis, consisting of a red, painful eye together with a 1-mm hypopyon and vitreous haze and cells.

\section{References}

1 Hooper CY, et al: Adjunctive antibiotics in the treatment of acute bacterial endophthalmitis following cataract surgery. Acta Ophthalmol 2012, DOI: 10.1111/j.1755-3768.2011.02365.x.

$\checkmark 2$ McCannel CA: Meta-analysis of endophthalmitis after intravitreal injection of anti-vascular endothelial growth factor agents: causative organisms and possible prevention strategies. Retina 2011;31:654-661.

-3 Moreau-Gaudry V, et al: Three cases of post-cataract surgery endophthalmitis due to Rhizobium (Agrobacterium) radiobacter. J Clin Microbiol 2012;50:1487-1490.

4 Barkana Y, et al: Reduction of conjunctival bacterial flora by povidone-iodine, ofloxacin and chlorhexidine in an outpatient setting. Acta Ophthalmol Scand 2005;83:360-363.

$\checkmark 5$ Sheldon AT Jr: Antiseptic 'resistance': real or perceived threat? Clin Infect Dis 2005;40:1650-1656.

6 Jarral OA, et al: Should surgeons scrub with chlorhexidine or iodine prior to surgery? Interact Cardiovasc Thorac Surg 2011;12:1017-1021.

7 Stickler DJ: Susceptibility of antibiotic-resistant Gram-negative bacteria to biocides: a perspective from the study of catheter biofilms. J Appl Microbiol 2002;92(suppl):163S-170S.

-8 Ferguson AW, et al: Comparison of 5\% povidone-iodine solution against 1\% povidone-iodine solution in preoperative cataract surgery antisepsis: a prospective randomised double blind study. $\mathrm{Br} \mathrm{J}$ Ophthalmol 2003;87:163-167. 